\title{
Chinese-English-Yi Public Opinion Information Database Construction and Implementation
}

\author{
Y.Y. Yang ${ }^{1}$ B.Z. Li $i^{2}$, J.M. Wang ${ }^{2 \star}$, C.S. Yuan², R Lin \& G.M. Lu J.L. Wang ${ }^{3}$ \\ ${ }^{1}$ School of Electrical Information Engineering, Yunnan MinZu University, Kunming 650500, China \\ The University of Bath, the United Kingdom \\ Yunnan Province colleges minority language information processing engineering research center, \\ Yunnan MinZu University, Kunming, China
${ }^{2}$ School of Electrical Information Engineering, Yunnan MinZu University, Kunming 650500, China Yunnan Province colleges minority language information processing engineering research center, Yunnan MinZu University, Kunming, China \\ ${ }^{3}$ Institute of Information Engineering, Weifang Vocational College of Engineering, Weifang, China \\ *Corresponding author: J.M. Wang
}

KEYWORD: Yi; Yi vocabulary text library; Yi language information technology;

\begin{abstract}
Language is an intangible cultural heritage of the human, it is more precious and longer than the tangible heritage history. Language resources preservation, research, development and utilization has become an important part of national resources. Through the research study of existing technologies and related systems, combined with ancient materials and Yi characteristics, this article study Chinese-English-Yi trilingual public opinion information corpus. This paper use a large number of instances (nearly three thousand words) to study Yi phonetic, lexical and grammatical characteristics and its corresponding relationship with Chinese and English, to optimize the public opinion information database and to establish a high quality of Chinese-English-Yi word text library. This paper used the existing recording equipment to build small Yi library as corpus base.
\end{abstract}

\section{INTRODUCTION}

China is a united multi-ethnic country. 56 ethnic groups create rich and colorful cultural heritage in the long-term production and life practice (Chang baby, 2002), which is the link emotional bond and maintain national unity on the basis of the country. Yi is one of the nation with a long history and ancient culture (Dai Qing Ha forward, 2005), is also China's sixth largest ethnic minority, mainly live in southwest China's Yunnan, Sichuan, Guizhou and Guangxi provinces (areas).

Browser/ServerYi language belongs to the Sino-Tibetan Tibeto-Burman Yi branch, there are six kinds of dialects. With the IPA (Hompson, Henry S, 1991) (Meng Zunxian et al, 1991) in 47 consonants, 12 vowels, 3 tone and a tight sound symbols, can accurately record the Yi language (Minnis, Stephen, 1991). The total population of China using the Yi language more than 650 million, with a population of more than 400 million in Yunnan Province (Nirenburg, S.ed, 1987), accounting for $65 \%$ of the total population. The six major dialect areas of Yi are distributed in Yunnan, Yuxi, Honghe Hani nationality autonomous prefecture and Chuxiong Yi nationality autonomous prefecture and Zhaotong city is the largest gathering area (Wang Jiamei, 2007) (Wang Jiamei, 2008) (Wang Jiamei, 2006).

To rescue this national cultural treasures of $\mathrm{Yi}$ as soon as possible, intelligent information processing in Chinese and English Yi trilingual should be strengthened (Yu, Shiwen. 1993). Such as building Yi language speech database, strengthen public opinion in Yi repository construction, strengthen the Yi Textual information processing research technology to accelerate cross-border ethnic culture (Yue Tao, 2005). Strengthening of Yi comprehensive national study to show, spread advanced culture, and promote the development of Chinese information processing field has important academic value (Zhao Tiejun, 2000) (Zhang Pu, 2005). 


\section{THE CONSTRUCTION METHOD OF YI, CHINESE, ENGLISH PUBLIC OPINION IN- FORMATION TRILINGUAL CORPUS.}

In this paper, the construction of high-quality Yi, Chinese, English trilingual online public opinion information corpus as the overarching goal, adhere language resource library construction and the combination of software development tools, taking into account the corpus and the Yi sound resource database library building.

The selection of Yi, Chinese, English trilingual corpus. This paper mainly use the political, legal, economic, scientific, cultural, educational and other language information as corpus basis from the Yunnan Nationalities University and Yunnan Province minority language information processing universities laboratory. We also use the browser / Server(B/S) three-tier system, WWW browser technology, combined with the JavaScript language, implements an online Chinese - English - Yi public opinion a voice message library that implements the original need for complex technology to achieve powerful, and save development costs.

The development and planning of Yi, Chinese, English public opinion information trilingual corpus. The core task of building of Yi, Chinese and English public opinion information is the organized corpus. In order to better carry out this work to ensure the quality and size of the corpus, a relatively complete, easy to operate corpus build process is the most important. Thus, through the corpus processing and handling, the corpus to analyze organizational issues such as the formation of a public opinion information trilingual corpus build process models, shown in Figure 1.

From the corpus collection of the Yi, English and Chinese, close to 50,000 corpus finished, a small parts as follows:

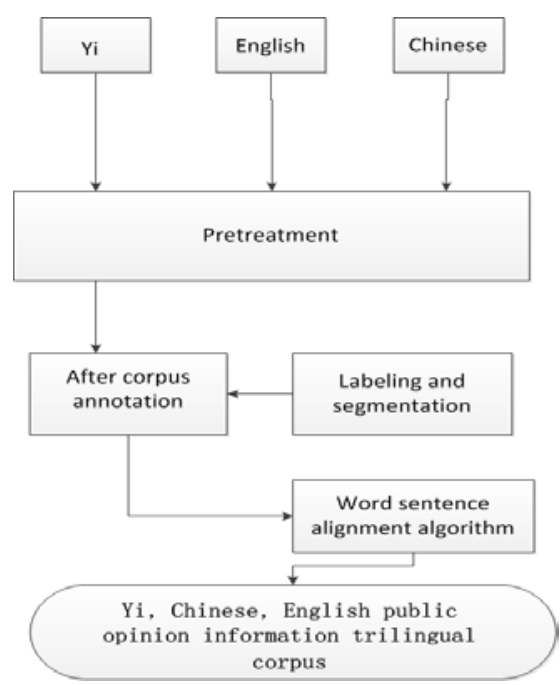

Figure 1. Yi-Chinese-English multilingual corpus implementation flow chart

From the corpus collection of the Yi, English and Chinese, close to 50,000 corpus finished, a small parts as follows:

Table 1. Chinese-Yi-English

\begin{tabular}{|c|c|c|}
\hline Chinese & $\mathrm{Yi}$ & English \\
\hline 钟爱 & gd & Love \\
\hline 偏爱 & $g \subset x$ & Preference \\
\hline 阻碍 & $N \bar{X}_{1}$ & Hinder \\
\hline 挂碍 & 为斗 & Obstruction \\
\hline 违碍 & $\mid \tilde{E} N$ & Taboo \\
\hline 干碍 & $\lambda \asymp$ & Prevent \\
\hline 妨碍 & $\lambda \asymp$ & Hinder \\
\hline 心理障碍 & $\int N \mathbb{N}^{*}$ & The psychological barrier \\
\hline 心里障碍 & $\sqrt[N]{N} * \mathbb{*}$ & Heart disorder \\
\hline 碍手碍脚 & 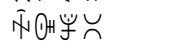 & Underfoot. \\
\hline 无障碍设施 & 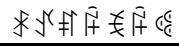 & Wheelchair Accessible \\
\hline
\end{tabular}

Yi language database design principles. In Chinese - English - Yi in the design of the online public opinion voice database should follow the following principles: The structure of the principles of openness, including open access permission of openness and content and structural principle. In- 
formation should fully consider the needs of the standardized, structured, will be the component part of online public opinion voice information database, module according to certain standard split, design, and production, eventually become a complete system. Second to consider friendly interface principle, principle of maintainability.

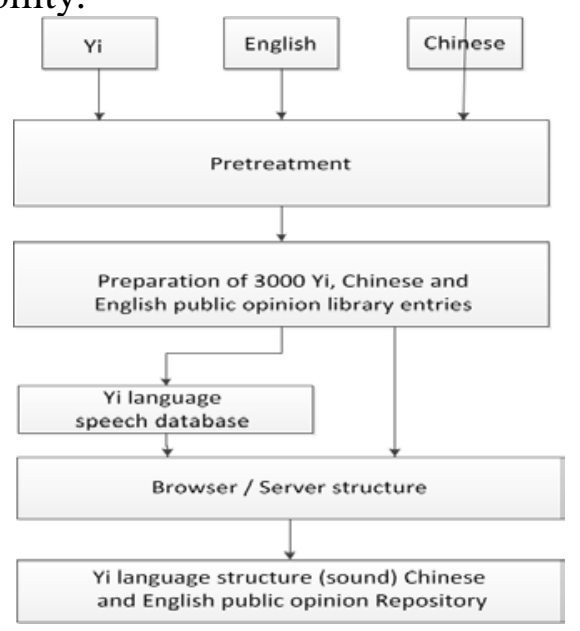

Figure 2. Yi language database design

\section{THE VOICE INFORMATION DATA BASE PROGRAM SYSTEM BUILDING OF CHI- NESE - ENGLISH - YI}

System development program. The online voice information database construction of Chinese English - Yi should ensure the efficient performance and strong public sentiment shared repository: Software system construction techniques: Browser / Server structure; The mature technology of browser , combined with Javascript language; Use the HTML scripting language; Picture made into JPG or GIF format files; The information of record processed into MPG format files. In the B/S architecture system, the user through the browser to request distribution in many servers on the network server to the browser's request for processing, the user information needed to return to the browser. While the rest such as data request, the processing, and the results back and dynamic Web page generation, such as access to the database and application execution work was done by the Web Server. B/S structure of the network structure as shown in figure 3:

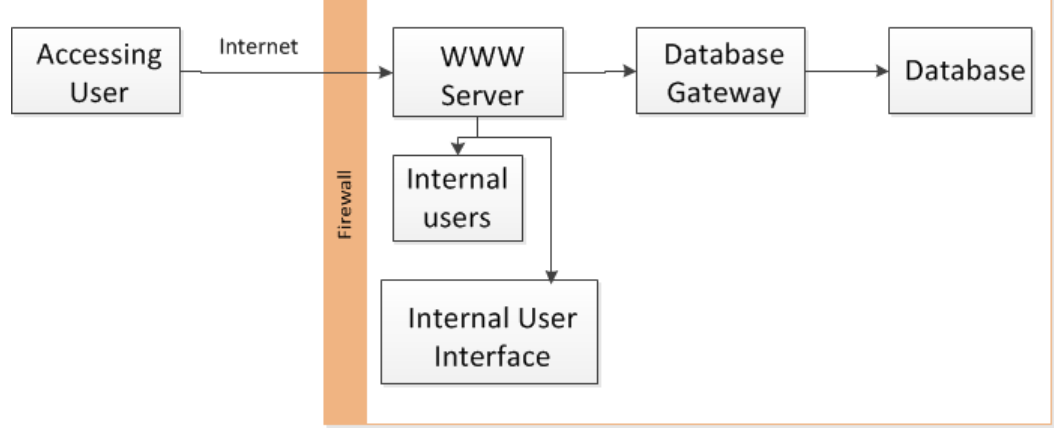

Figure 3. Network structure

\section{CHINESE - ENGLISH - YI ONLINE VOICE REPOSITORY OF PUBLIC OPINION}

In this paper, the client interface design and development process, using Ajax + JSP + Struts structure. In the business logic layer, using Spring + JavaBean structure, process the query, the results of the conversion, dynamic word matching functions. In the data storage layer using a relational database structure Hibernate audio file. Users get relevant results in the query text also can hear the standard pronunciation examples. The following figure 4 for Online Chinese - English - Yi online repository of public opinion a voice interface: 


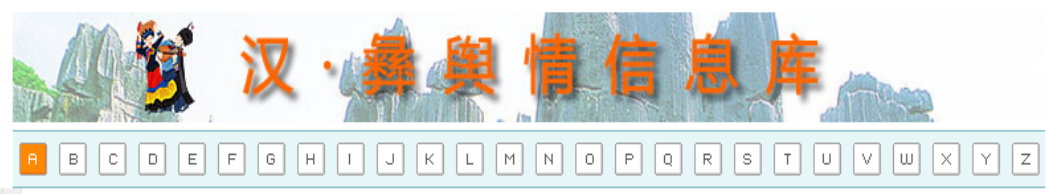

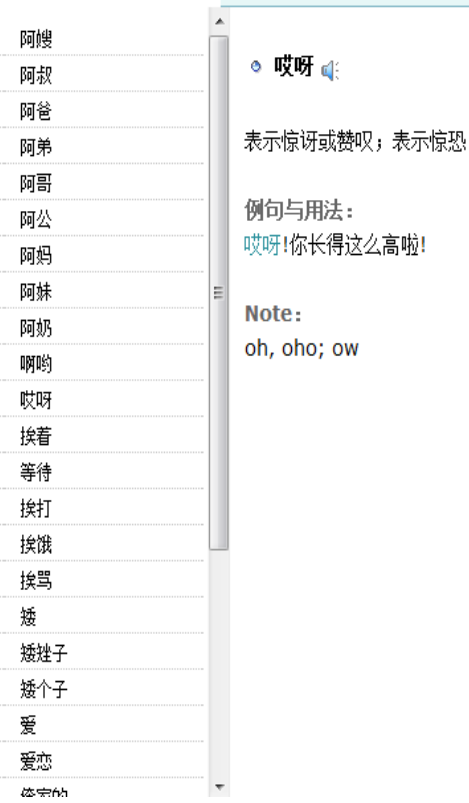

Figure 4. Online Chinese - English - Yi public opinion Voice Interface Repository

Database technology. Using free and efficient MySQL relational database software, not only to meet all the needs of the system, and can greatly reduce application costs.

Application server technology. Using a free, efficient, widely used Tomcat application server as the Web server system and JavaBean \& Servlet container at the same time meet the need to use the system, reducing the cost of the application.

Instructions for use. After the site is running, use the browser to access the Web site address, on the left click on the search terms the first word, easy to instantly displays search results right, click on the specific terms, the right of the main screen will display entries detailed interpretation, including: entry name, IPA pronunciation examples buttons, Chinese Interpretation, English interpretation, click pronunciation example button, you can hear the human voice demo.

\section{CONCLUSION}

This article uses the Browser/Server three-tier architecture system, WWW Browser technology, combined with the JavaScript language, to yi trilingual public opinion information chinese-english corpus research, build implemented an online public opinion - English - yi han language speech database. Yunnan is a multi-ethnic, language, and province, this paper is a case, as the study of minority languages and cross-border ethnic language to the comprehensive and thorough, its development value, the demonstration value, the theory of value is worth attention.

\section{ACKNOWLEDGEMENTS}

This work was financially supported by the National Natural Science Foundation of China (61363085), Major projects in Yunnan Province (ZD2013013), Southeast Asia West Asia Research Center of Yunnan Province tender subject of Yunnan MinZu University (DY2014YB01), High level construction of university scientific research project of Yunnan MinZu University. Yunnan MinZu University Graduate Innovation Fund focused research project, State Language Commission major research projects:" Yuan said information and extraction method of the minority language and culture", WT125-61, Weifang Engineering Vocational College research projects (WGZZ201409, WGZZ201410). 


\section{REFERENCES}

[1]. Chang baby. "Bilingual Corpus-based Translation Equivalent automatic access to research." 2002 (2).

[2]. Dai Qing Ha forward. Naniwa language research [M]. Ethnic Publishing House, 2005.1. [15]Ifrim G,Theobald M,and Weikum G.Learning word-to-concept mappings for automatic text classification[J].ICML Workshop on Learning in Web Search,2005.

[3]. Hompson, Henry S. 1991. "Automatic Evaluation of Translation Quality: Outline of Methodology and Report on Pilot Experiment" in Falkedal ed. Proceedings of the Evaluator's Forum. Geneva: ISSCO. PP215 224.

[4]. Meng Zunxian. Fang Bolong Han Dai dictionary. Yunnan Nationalities Publishing House. 1991.6.1.

[5]. Minnis, Stephen. 1991. "Constructive Machine Translation Evaluation" in Falkedal ed. Proceedings of the Evaluator's Forum. Geneva: ISSCO. PP99 116.

[6]. Nirenburg, S.ed. 1987. Machine Translation: Theoretical and Methodological Issues. Cambridge: Cambridge University Press. PP28 29.

[7]. Sino-Tibetan cognates studies (a) Demonstrated, Dingbang new editor Guangxi Ethnic Publishing House in September 2000.

[8]. Tibeto-Burman language studies Dai Qing Ha of Yunnan Nationalities Publishing House in December 1998.

[9]. Wang Jiamei, for natural language understanding Burman branch of Chinese phonetic system research, scientific era, 2007.12.

[10]. Wang Jiamei, image segmentation based on the ancient Yi character recognition system research, Journal of Natural Science, Yunnan Nationalities University, 2008.14 (1).

[11]. Wang Jiamei, Yi Ancient Digital Research - Yi Language Ancient special website, college, 2006.07: 149-150.

[12]. Yue Tao "machine translation of the status quo and development trend." Computer Education 2005.4 p72-74.

[13]. Yunnan, Sichuan and Guizhou and Guangxi Yi text set by Yunnan, Guizhou, Sichuan Ethnic Publishing House jointly published three .2001.

[14]. Yu, Shiwen. 1993. "Automatic Evaluation of Output Quality for Machine Translaton" in Arnold, Doug \& R. Lee Humphreys et al. eds. Machine Translation: Special Issue on Evaluation of MT Systems, 8 (1-2). Dordrecht: Kluwer Academic Publishers. PP117 124.

[15]. Zhang Pu open. Diannan Yi language dictionaries. Yunnan Nationalities Publishing House. 2005.10 .

[16]. Zhao Tiejun other "machine translation principle" Harbin: Harbin Institute of Technology Press, 2000. 